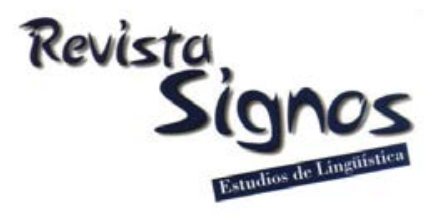

\title{
Alfabetización académica y pedagogía de género discursivo en la Lingüística Sistémico Funcional. Una experiencia de trabajo
}

\author{
Academic literacy and discourse gender pedagogy in Systemic \\ Functional Linguistics. A classroom experience
}

\author{
Ilene Rojas García \\ UNIVERSIDAD DEL NORTE \\ COLOMBIA \\ zellene7@gmail.com
}

\author{
Giohanny Olave \\ Arias \\ UNIVERSIDAD DE BUENOS AIRES \\ ARGENTINA \\ olavearias@gmail.com
}

\author{
Mireya Cisneros \\ Estupiñán \\ UNIVERSIDAD TECNOLÓGICA DE \\ Pereira \\ COLOMBIA \\ mireyace@gmail.com
}

Recibido: 01/XII/2015 / Aceptado: 18/V/2016

\section{Resumen}

Se analiza una experiencia de aula para enseñar lectura y escritura académicas. La experiencia estuvo orientada hacia el desarrollo de la competencia argumentativa a través de textos de opinión, tomando como base teórica y metodológica la Pedagogía de Género, derivada de la Lingüística Sistémico-Funcional. La propuesta parte del conocimiento de los géneros discursivos y privilegia el trabajo conjunto y la visión del docente como mediador. Durante la intervención, se puso en juego los saberes previos, el sentido social del texto, la delimitación del campo, tenor y modo y el análisis de las formas del lenguaje al servicio de la intención comunicativa. Desde allí, quedaron en evidencia diversos problemas que persisten en los estudiantes a nivel de la lectura y la escritura: las insuficiencias en el conocimiento previo, la tensión análisis / síntesis, la transferibilidad del análisis, la dimensión argumentativa del género comentario, la extensión textual, la gestión de las fuentes y la construcción del otro. Concluimos que la introducción de problemáticas sociopolíticas a través de los textos es importante para la planificación de las secuencias didácticas, en tanto que van dirigidos hacia la formación de ciudadanos desde el derecho a la palabra, la pluralidad de puntos de vista, la participación democrática y la coexistencia en el disenso, en los textos escritos.

Palabras Clave: Lectura crítica, escritura académica, competencias comunicativas, formación profesional. 


\begin{abstract}
We analyzed a classroom experience to teach academic reading and writing. The experience was oriented towards the development of the argumentative competence through opinion texts. The theoretical and methodological framework we have adopted is that of Genre Pedagogy, derived from the Systemic Functional Linguistics. This framework is based on discourse genres, that is, it favors joint work, and conceives the teacher as a mediator. During the intervention process, we took into consideration prior knowledge, social meaning of the text, the delimitation of the field, tenor and mode, and the analysis of the forms of language in the communicative event. From this analysis, many problems in students' reading and writing level were evidenced. They are: the deficiency in prior knowledge, analysis/synthesis tension, the analysis transferability, the argumentative dimension of the commentary genre, the length of the texts, and the management of sources and the construction of the other. Thus, we conclude that the introduction of sociopolitical issues textually presented are important for the planning of teaching programs as long as they lead to the education of citizens having the right to speak from a plurality of points of view, democratic participation, and the coexistence in dissent in written texts.
\end{abstract}

Key Words: Critical reading, academic writing, communicative competences, professional training.

\title{
INTRODUCCIÓN
}

Analizamos la experiencia en el desarrollo del segundo de dos cursos universitarios orientados al mejoramiento de habilidades en lectura y escritura académica, para el uso del lenguaje propio de la educación superior, la ciencia y los espacios de ejercicio ciudadano y profesional ${ }^{1}$. El primer curso se enfoca en la comprensión y producción de textos académicos expositivos, principalmente, los resúmenes y mapas conceptuales. En el segundo, se busca mejorar la lectura y la producción de textos académicos argumentativos. Ambos tienen una orientación teórico-práctica y el énfasis especial en el desarrollo de la autonomía, la solidaridad (en el sentido del aprendizaje entre pares) y la criticidad (Universidad del Norte, 2015).

La base teórica sobre la cual se sustentan institucionalmente estos espacios de formación es la Pedagogía de Género (Martin \& Rose, 2012), desde donde se promueve tanto el estudio del texto como la intención de formar lectores y escritores autónomos. Se trata de una propuesta teórica y práctica que parte del conocimiento de los géneros discursivos desde la LSF, que se integra con los enfoques metodológicos propuestos por Vygotsky $(1978,1998)$ y Bruner $(1963,1983)$, en donde se privilegia el trabajo conjunto y la visión del docente como mediador.

Concretamente, en el presente documento analizamos una experiencia de aula orientada a la lectura y la escritura de textos de opinión en el segundo curso universitario. 
En el mencionado curso, se identificó la forma como se organizan los textos argumentativos desde sus diferentes elementos estructurales, la función de cada componente regular, el tipo de valoraciones que aparecen y los puntos de vista que dialogan. De este modo, durante la intervención, se buscó que el estudiante lograra una apropiación conceptual de las formas y usos académicos de la lengua, para producir textos similares.

El desarrollo del curso involucró la reflexión en torno a la especificidad temática y social de los textos a leer y escribir, para enfatizar en la forma como estos pueden ayudar a movilizar reflexiones sobre el ámbito político nacional. En este sentido, se propuso el estudio de textos que tuvieran pertinencia social en una coyuntura histórica particular colombiana: el proceso de paz que adelanta desde 2012 el gobierno colombiano con el grupo guerrillero autodenominado Fuerzas Armadas Revolucionarias de Colombia, FARC, tras seis décadas de conflicto armado interno (CHCV, 2015; GESCAL, 2015).

La posibilidad de finalizar el conflicto a través de un proceso de paz es una temática que permite partir de dos principios epistemológicos: 1) la vinculación directa con los procesos de comprensión y producción textual, toda vez que los textos no son objetos aislados de sus realidades sociohistóricas particulares, y de hecho, contribuyen a construirlas (Bajtín, 1982); y 2) interpela a toda la población colombiana, específicamente, al ámbito educativo de la formación de profesionales en cualquier área del saber.

La temática, además, permite vincular la lectura y la escritura universitaria con la formación de intereses, habilidades y necesidades de la participación democrática, la discusión de puntos de vista divergentes y el esfuerzo de enseñar a coexistir en la diferencia a partir de la apertura a la polémica como modalidad argumentativa (Plantin, 2011; Amossy, 2014). Aquí no se trata de desplazar 'lo lingüístico' por 'lo ético', ni de moralizar las prácticas de alfabetización académica, sino de involucrarlas en la responsabilidad formativa de la universidad (Bolívar \& Parodi, 2015) en relación con aspectos sociales como la indiferencia frente a fenómenos políticos coyunturales, o bien, la polarización radical de opiniones en torno a ellos, que reproducen modos de exclusión y de violencia. En este sentido, el estudio del género:

"constituye una constelación de potencialidades de convenciones discursivas, sustentada por los conocimientos previos de los hablantes/escritores y oyentes/lectores (almacenados en la memoria de cada sujeto), a partir de constricciones y parámetros contextuales, sociales y cognitivos" (Parodi, 2008: 26).

La experiencia que aquí se revisa presenta las posibilidades de la Pedagogía de Género, como base para el desarrollo de la comprensión y producción de textos argumentativos. 


\section{Marco de referencia}

La Pedagogía de Género, desde la Lingüística Sistémica Funcional (LSF) (Halliday, 2014), sostiene que antes de que el género pueda materializarse como signo lingüístico, se establecen una serie de conexiones previas. De afuera hacia adentro, está el nexo entre el contexto y el texto, materializándose en el registro. En este estrato están las dimensiones de campo, tenor y modo. El campo, se refiere a lo que sucede en el contexto a nivel de: 1) la naturaleza de la actividad social y semiótica; y 2) el dominio de la experiencia con la cual se relaciona la actividad. El tenor involucra a los participantes de la situación; aquí son relevantes los roles de los actores (institucionales, de estatus, de cercanía o de escala sociométrica) y los valores que los actantes incluyen en la interacción (ya sea neutro o cargado, positiva o negativamente). El modo trata el rol del lenguaje u otros sistemas semióticos en la situación: la división de la labor entre las actividades semióticas y las sociales (el lenguaje constituye o apoya la actividad social), entre las actividades semióticas y las lingüísticas, el modo retórico (informar, explicar, proponer, etc.), el turno (dialógico o monológico), el medio (escrito o hablado) y el canal (fónico o gráfico). El registro permite explicar la forma como las prácticas sociales determinan el uso de recursos lingüísticos.

Estas dimensiones están en el contexto de situación y en la función misma del lenguaje. Al internalizar las relaciones entre el contexto de situación y el texto se ponen en evidencia las metafunciones del lenguaje (Halliday, 2014). La metafunción ideacional permite construir la experiencia de la vida social, interpretar y representar el mundo real y el mundo interior, así como establecer relaciones lógicas; en este sentido, el lenguaje organiza la experiencia y ayuda a construir la visión del mundo. La función interpersonal permite establecer relaciones entre los hablantes, interactuar con otros, asumir roles y presentar actitudes, sentimientos, juicios, deseos o creencias. La función textual transforma las relaciones con el mundo y con los otros, en significado discursivo. A través de las opciones que brinda el lenguaje es posible establecer relaciones de coherencia entre las cláusulas de una emisión lingüística. El género discursivo es definido desde este enfoque, por Martin y Rose (2012: 54), como un:

“(...) proceso social, orientado a objetivos y realizado en etapas; social porque inevitablemente estamos tratando de comunicarnos con otros (incluso si ellos no leen o responden inmediatamente a nuestro trabajo), orientado a objetivos porque siempre tenemos un propósito al escribir y nos sentimos frustrados si no lo cumplimos, y realizado en etapas en tanto que usualmente nos toma más de un paso alcanzar nuestros objetivos".

El género atraviesa varios estratos: el primero es el de la semántica, corresponde al paso de la experiencia de las relaciones interpersonales que es transformada en lenguaje. Posteriormente, el significado se convierte en palabras en el estrato de lo 
lexicogramatical (Halliday, 2014). La interpretación de esta secuencia interrelacionada de estratos puede ser transferida al campo de la alfabetización académica en doble vía: hacia arriba, al momento de enfrentarse a una lectura en la que se parte del material grafológico para inferir de allí los procesos sociales que lo configuran; y hacia abajo, en tanto que se parte de un requerimiento social para producir un texto. Desde la Pedagogía de Género, reconocer los aspectos de la lengua a nivel genérico facilita y optimiza su transferencia y aplicación en entornos educacionales y permite trabajar desde la conciencia funcional de las elecciones lingüísticas.

Martin y Rose (2003: 8) resaltan el carácter social del género al presentarlo como "los diferentes tipos de texto que difunden distintos tipos de contextos sociales". Estos géneros son aprendidos en la interacción, y sus particularidades se constituyen como modelos de uso desde las conversaciones cotidianas, hasta las esferas del lenguaje científico o político. La descripción de los géneros se realiza a partir de la relación que se establece entre ellos, pues las diferencias ayudan a definir de qué género se trata. En este sentido, los géneros pueden relacionarse con otros a partir de sus dimensiones de carácter individual o con participantes genéricos, por su intención narrativa, explicativa o argumentativa (Gee \& Handford, 2012).

La Pedagogía de Género ha diseñado aspectos metodológicos que ayudan a los estudiantes a comprender el contexto cultural, las etapas y las herramientas del lenguaje de los textos estudiados. Martin y Rose (2012), en el proyecto Reading to Learn, presentan la secuencia didáctica como un ciclo guiado por el maestro: inicia con la selección de un género y la reflexión sobre el campo del saber en el que se inscribe; luego pasa por la deconstrucción, allí se analizan las relaciones entre el campo, el tenor y el modo, como mecanismo de acción en el contexto cultural. Una vez comprendidos estos aspectos, se pasa al ejercicio de escritura, conservando el mismo género elegido. Este ejercicio parte de un trabajo en conjunto, modelado por el docente, para dar paso al trabajo independiente; en este último, el estudiante puede emular tanto las características genéricas del texto abordado, como las estrategias que fueron utilizadas por el maestro en la construcción conjunta. Finalmente, se propone generar espacios de discusión que valoren los textos a nivel de sus posibilidades de análisis de la teoría, la práctica y la ideología (Figura 1). 


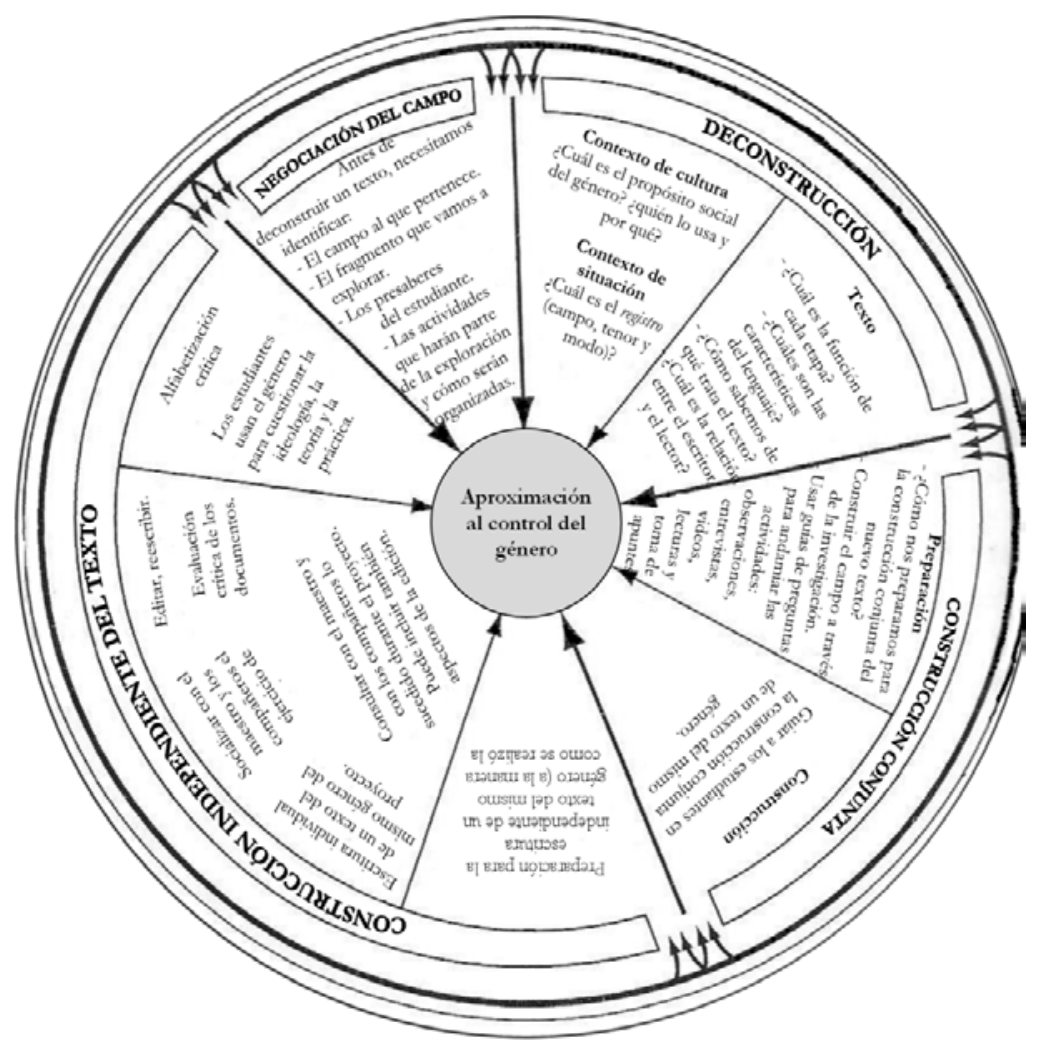

Figura 1. Ciclo de enseñanza y aprendizaje del proyecto de Lenguaje y Poder Social (Martin \&Rose, 2012: 65). Traducción propia.

Este modelo ha sido utilizado como base para otras investigaciones, en busca de adaptaciones didácticas para la lectura y la escritura en la educación superior a través del currículo, es decir, relacionándola directamente con los textos que se leen y se escriben para aprender en las distintas disciplinas científicas (Marinkovich \& Morán, 1998; Bazerman, Bonini \& Figueiredo, 2009; Natale, 2012; Moyano, 2014). En el ámbito educativo, la Pedagogía de Género ha contribuido en la descripción de textos que cumplen con objetivos didácticos (aún de manera indirecta) (Martin \& Rose, 2008). Tales estudios permiten direccionar la enseñanza de la lengua hacia objetivos reales de comunicación, desarrollo del pensamiento y uso del lenguaje.

\section{Metodología}

Se realizó una intervención didáctica durante dos meses en clases de tres horas semanales, con 160 estudiantes de segundo semestre, divididos en cinco grupos que cursan Competencias Comunicativas II y pertenecen a diferentes programas académicos ofrecidos por la Universidad del Norte. Los registros de observación se realizaron en diarios de campo a cargo del docente principal y de un observador externo al desarrollo de la clase. Asimismo, se recolectaron los textos producidos por 
los estudiantes, tanto a nivel de las tareas de comprensión textual como de producción escrita, según las consignas estipuladas en la secuencia didáctica.

La intervención didáctica siguió las orientaciones metodológicas de la Pedagogía de Género (Martin \& Rose, 2005, 2008, 2012) de la LSF en relación con los estratos del lenguaje. Aquí interesa conseguir un conocimiento suficiente de la lengua en uso para democratizar el saber. El ciclo de enseñanza de Martin y Rose (2012) enfatiza en los aspectos del contexto de cultura y el contexto de situación, que enmarca el análisis de campo, tenor y modo. Se resaltaron cuatro aspectos en el estudio del texto: 1) La construcción del saber nuevo sobre la base de los conocimientos ya elaborados por los estudiantes, buscando establecer conexiones significativas y allanando el camino para la elaboración de hipótesis previas a la lectura (negociación del campo); 2) La ubicación del texto en un contexto real de uso con intenciones particulares, con el fin de comprender el sentido social que ubica el texto como parte fundamental en el engranaje social (contexto de cultura); 3) La delimitación de la realidad en la que se inscribe el texto (campo), las relaciones de poder que se establecen entre los participantes (tenor) y las estrategias lingüísticas usadas para llevar a cabo la intención comunicativa (modo): 4) El análisis de las formas del lenguaje al servicio de la intención comunicativa. Estos aspectos se encuentran directamente relacionados con la materialidad textual y se pueden encontrar los recursos necesarios para adelantar la 'deconstrucción'; pero también tiene un papel fundamental el conocimiento previo del lector sobre el tema. En este sentido, se generó un movimiento de ida y vuelta entre el texto y la experiencia social, que fue tejiendo la dimensión discursiva e inscribió el texto en un género particular. Así, en un intento por evitar posibles confusiones desde el dominio del metalenguaje, y con el objetivo de resaltar la función social de los textos objeto de análisis, se procuró mantener los cuatro aspectos mencionados en su estudio, pero enfocándolos en la conceptualización sobre género elaborada por Martin y Rose (2012).

En la Tabla 1, se puede observar los diferentes momentos de la secuencia didáctica, se relacionan las consignas de trabajo en el orden de la secuencia didáctica propuesta y se vinculan con la actuación del docente y los materiales utilizados:

Tabla 1. Proceso de intervención didáctica.

\begin{tabular}{|c|c|c|c|}
\hline $\begin{array}{l}\text { Intervención del } \\
\text { docente }\end{array}$ & Ejercicios propuestos & Materiales & $\begin{array}{c}\text { Ubicación en el } \\
\text { ciclo (Martin \& } \\
\text { Rose, 2012) } \\
\end{array}$ \\
\hline \multicolumn{4}{|c|}{ LECTURA } \\
\hline $\begin{array}{l}\text { 1. Introducción } \\
\text { comentada sobre el } \\
\text { propósito social de los } \\
\text { textos argumentativos } \\
\text { y la participación } \\
\text { ciudadana en la } \\
\text { democracia }\end{array}$ & $\begin{array}{l}\text { 1. Visualizado grupal de una } \\
\text { conferencia de Jaime Garzón } \\
\left(1997^{2}\right) \\
\text { 2. Diálogo grupal en torno a la } \\
\text { audiencia, el contexto social de } \\
\text { producción, el propósito, las } \\
\text { estrategias argumentativas }\end{array}$ & $\begin{array}{l}\text { Conferencia } \\
\text { en } \\
\text { audiovisual. }\end{array}$ & $\begin{array}{l}\text { Negociación del } \\
\text { campo. }\end{array}$ \\
\hline
\end{tabular}




\begin{tabular}{|c|c|c|c|}
\hline colombiana. & $\begin{array}{l}\text { utilizadas y la representación } \\
\text { de los jóvenes y de la } \\
\text { universidad en el video } \\
\text { visualizado. }\end{array}$ & & \\
\hline $\begin{array}{l}\text { 2. Lectura a viva voz } \\
\text { del texo "Sí al proceso } \\
\text { de paz" (Prado, 2015). }\end{array}$ & $\begin{array}{l}\text { 1. Análisis modelado de } \\
\text { campo, tenor y modo en el } \\
\text { texto. }\end{array}$ & $\begin{array}{l}\text { Artículo de } \\
\text { opinión } \\
\text { favorable al } \\
\text { proceso de } \\
\text { paz. }\end{array}$ & \multirow[t]{4}{*}{ Deconstrucción. } \\
\hline $\begin{array}{l}\text { 3. Lectura a viva vOZ } \\
\text { del texto "Y siguen en } \\
\text { Katamarán" } \\
\text { (Kertzman, 2015). }\end{array}$ & $\begin{array}{l}\text { 1. Análisis modelado de } \\
\text { campo, tenor y modo en el } \\
\text { texto. } \\
\text { 2. Modelo de comparación con } \\
\text { el texto anterior. }\end{array}$ & $\begin{array}{l}\text { Artículo de } \\
\text { opinión } \\
\text { desfavorable } \\
\text { al proceso de } \\
\text { paz. }\end{array}$ & \\
\hline $\begin{array}{l}\text { 4. Mediación en } \\
\text { lectura individual y } \\
\text { grupal de los textos: } \\
\text { "Les importan un } \\
\text { pimiento" } \\
\text { (Hernández, 2014) y } \\
\text { "Nada es fácil" } \\
\text { (Duzán, 2015). Y de } \\
\text { los comentarios de los } \\
\text { lectores en el espacio } \\
\text { virtual. }\end{array}$ & $\begin{array}{l}\text { 1. Análisis individual de } \\
\text { campo, tenor y modo en los } \\
\text { textos. } \\
\text { 2. Valoración de los } \\
\text { comentarios de los internautas } \\
\text { en el espacio virtual. } \\
\text { 3. Discusión grupal de los } \\
\text { hallazgos. }\end{array}$ & \multirow[t]{2}{*}{$\begin{array}{l}\text { Artículos de } \\
\text { opinión y } \\
\text { comentarios } \\
\text { de los } \\
\text { internautas, } \\
\text { favorables y } \\
\text { desfavorables } \\
\text { al proceso de } \\
\text { paz. }\end{array}$} & \\
\hline $\begin{array}{l}\text { 5. Asesoría de grupos } \\
\text { de trabajo para el } \\
\text { análisis de los textos } \\
\text { "El proceso de paz en } \\
\text { Colombia (I)" (Roa, } \\
\text { 2014) o } \\
\text { "El desescalamiento } \\
\text { es un clamor nacional } \\
\text { e internacional" } \\
\text { (Timochenko, 2015). }\end{array}$ & $\begin{array}{l}\text { 1. Elección de uno de los } \\
\text { textos propuestos para el } \\
\text { trabajo en grupos. } \\
\text { 2. Análisis de campo, tenor y } \\
\text { modo. }\end{array}$ & & \\
\hline \multicolumn{4}{|c|}{ ESCRITURA } \\
\hline $\begin{array}{l}\text { 1. Orientación en el } \\
\text { manejo de un foro } \\
\text { virtual institucional. } \\
\text { Diagnóstico de la } \\
\text { escritura de los } \\
\text { estudiantes. }\end{array}$ & $\begin{array}{l}\text { 1. Participación en un foro } \\
\text { virtual sobre el Proceso de } \\
\text { Paz. }\end{array}$ & $\begin{array}{l}\text { Espacio de } \\
\text { foro virtual. }\end{array}$ & \multirow[t]{2}{*}{$\begin{array}{l}\text { Construcción } \\
\text { conjunta. }\end{array}$} \\
\hline $\begin{array}{l}\text { 2. Modelado de } \\
\text { producción de } \\
\text { argumentos para el } \\
\text { género comentario del } \\
\text { lector. }\end{array}$ & $\begin{array}{l}\text { 1. Búsqueda bibliográfica } \\
\text { sobre el proceso de paz. } \\
\text { 2. Uso de los hallazgos } \\
\text { bibliográficos para } \\
\text { fundamentar opiniones } \\
\text { asumidas y construir } \\
\text { argumentos. } \\
\text { 3. Escritura de borradores de } \\
\text { argumentos. }\end{array}$ & $\begin{array}{l}\text { Páginas web } \\
\text { académicas e } \\
\text { informativas. } \\
\text { Modelo de } \\
\text { estructura de } \\
\text { un } \\
\text { argumento. }\end{array}$ & \\
\hline $\begin{array}{l}\text { 3. Explicación sobre } \\
\text { mecanismos de } \\
\text { participación virtual } \\
\text { en la web Mesa de } \\
\text { Conversaciones (2015) }\end{array}$ & $\begin{array}{l}\text { 1. Visualizado de tutorial sobre } \\
\text { participación en la web oficial } \\
\text { del Proceso de paz. } \\
\text { 2. Análisis de comentarios } \\
\text { disponibles en la web, desde el }\end{array}$ & $\begin{array}{l}\text { Video- } \\
\text { tutorial, } \\
\text { página web. } \\
\text { Formulario } \\
\text { con }\end{array}$ & $\begin{array}{l}\text { Construcción } \\
\text { independiente del } \\
\text { texto. }\end{array}$ \\
\hline
\end{tabular}




\begin{tabular}{|l|l|l|l|}
\hline del proceso de paz. & $\begin{array}{l}\text { campo, tenor y modo. } \\
\text { 3. Borradores de comentarios } \\
\text { para enviar al espacio web de } \\
\text { la Mesa de Conversaciones. }\end{array}$ & $\begin{array}{l}\text { directrices } \\
\text { para la } \\
\text { redacción y } \\
\text { envío del } \\
\text { comentario. }\end{array}$ & \\
\cline { 1 - 3 } $\begin{array}{l}\text { 4. Revisión de } \\
\text { borradores de } \\
\text { comentarios para la } \\
\text { Mesa de } \\
\text { Conversaciones. }\end{array}$ & $\begin{array}{l}\text { 1. Redacción de versiones } \\
\text { finales de comentarios para la } \\
\text { Mesa de Conversaciones. } \\
\text { 2. Envío de comentarios a la } \\
\text { página web. }\end{array}$ & $\begin{array}{l}\text { Formulario } \\
\text { con } \\
\text { directrices } \\
\text { para la } \\
\text { redacción y } \\
\text { envío del } \\
\text { comentario. }\end{array}$ & \\
\cline { 1 - 2 } $\begin{array}{l}\text { 5. Revisión colectiva y } \\
\text { coevaluación de los } \\
\text { comentarios } \\
\text { elaborados. }\end{array}$ & $\begin{array}{l}\text { 1. Juego de roles asumiendo el } \\
\text { papel de los personajes que } \\
\text { participan en los Diálogos de } \\
\text { paz. }\end{array}$ & $\begin{array}{l}\text { Escarapelas. } \\
\text { Rúbrica de } \\
\text { valoración. }\end{array}$ & \\
& $\begin{array}{l}\text { 2. Revisión de los comentarios } \\
\text { elaborados por los }\end{array}$ & \\
compañeros. & \\
\hline
\end{tabular}

\section{Resultados}

\subsection{El problema del conocimiento previo}

El desconocimiento del tema fue el impedimento con mayor incidencia tanto en la comprensión como en la producción textual. El conjunto de saberes previos sobre una temática social y política compleja determina los modos de establecer relaciones con los textos que se proponen y la mayor o menor profundidad de esas relaciones. En principio, el 38\% de los estudiantes desconocía los tópicos que aparecían en los textos propuestos, o bien, los relacionaban de manera muy general con la temática (el proceso de paz) que habían escuchado, pero de la que poco o nada habían leído ni escrito. El 43\% reconoció que los conocimientos de base provenían del sentido común y de las tradiciones ideológicas de sus medios sociales privados (familia) y públicos (medios masivos, redes sociales, etc.); en todo caso, se trataba de saberes no reflexivos, sin puntos de vista propios frente a asuntos de pertinencia social como la violencia y la paz en el país.

Con respecto a la comprensión, la mayor dificultad estuvo en la recuperación de la información implícita, particularmente de lo que Ducrot (2001) denomina sobreentendidos, es decir, aquellos datos no explícitos que se encuentran anclados débilmente a la materialidad de los enunciados y que desplazan la responsabilidad enunciativa del locutor hacia la interpretación que debe realizar el lector para comprender la voz o punto de vista del enunciador construido. Los sobreentendidos textuales difícilmente podían llenarse con los presaberes disponibles de los estudiantes, pues requerían reponer información vinculada con el sesgo ideológico de los periodistas y de sus plataformas de publicación. El no poder recuperar esos 
sentidos reduce la posibilidad de que cualquier lector tome una postura crítica frente a los planteamientos del autor; de ahí que el lector termina por adherirse a los puntos de vista al construirlos como enunciadores autorizados. Por ejemplo, el artículo de Prado (2015) argumenta que los colombianos deben 'tragarse muchos sapos' para lograr el tratado de paz. Esa construcción figurativa alude a las concesiones que le hace el gobierno a las FARC y que no son bien vistos por la ciudadanía en razón de la violencia que este grupo insurgente ha ejercido sobre la población civil. La expresión utiliza una figura del registro popular para eludir la gravedad de las concesiones otorgadas a la guerrilla y evitar especificarlas. Los estudiantes no alcanzan a recuperar el sentido implícito de la expresión utilizada por el periodista, ni las razones posibles de ese sobreentendido.

En lo que respecta a la escritura, solicitar un documento de opinión sobre una realidad con más de medio siglo de historia en la que han participado diversidad de actores sociales, requiere un conocimiento reflexivo de la temática, es decir, que se asuma una actitud de cuestionamiento frente a los textos leídos, busque otras fuentes, confronte puntos de vista y genere posturas que aporten a la discusión. El ejercicio a corto plazo estuvo enfocado más en la producción de textos con intervención social que en la formación de analistas políticos; pero se trata de escrituras que no desplacen su función social y democrática por el énfasis en el conocimiento estructural de lo que se escribe. Por tanto, en el ejercicio de escritura se valoró la capacidad de utilizar el conocimiento formal de la producción textual para participar con una voz propia en el proceso de paz, a través del espacio que la Mesa de Conversaciones dispuso en internet para que la ciudadanía enviara sus comentarios.

El escaso conocimiento previo de los estudiantes se manifestó en las primeras discusiones grupales sobre los artículos de opinión y en la participación en el foro virtual institucional que se planteó a manera de diagnóstico de la escritura de ese género. En el primer caso, fue necesario confrontar artículos que tuvieran opiniones encontradas para ayudar a desnaturalizar opiniones, reconocer los puntos de vista contrarios, reconocer estrategias argumentativas distintas y la pluralidad de actitudes frente al proceso de paz. En el segundo caso, el diagnóstico de la participación en el foro virtual institucional, permitió ver la superficialidad de los comentarios de quienes no buscaron construir argumentos a partir de una consulta previa pertinente y, además, la dificultad para demostrar el cruce de lecturas heterogéneas y la capacidad de ponerlas en juego en la elaboración de opiniones propias. Frente a este diagnóstico, se propuso una orientación extendida sobre consulta bibliográfica, mecanismos de introducción del discurso referido, tipos de citación y construcción de relaciones lógicas entre el discurso ajeno y el propio.

Otro tipo de saber previo que no está referido a la temática social propuesta, sino al contenido lingüístico es el conocimiento del género que denominamos 'comentario 
del lector en espacio virtual'. El diagnóstico dejó ver, por un lado, el desempeño intuitivo de los estudiantes en la producción de este género que no les es extraño, dada su presencia cada vez mayor en las páginas web informativas y, particularmente, en las redes sociales. Por otro lado, se evidenció la inestabilidad del género en cuanto a sus regulaciones y regularidades que algunos trabajos en análisis del discurso vienen atendiendo con mucho interés (Amossy, 2011; Acosta, 2012; Acebedo, 2014).

\subsection{El problema de la tensión análisis / síntesis en la lectura}

El ciclo de enseñanza y aprendizaje del Proyecto de Lenguaje y Poder Social (Martin \& Rose, 2008), presentado en la metodología, ofrece una serie de preguntas y consignas que permiten direccionar la intervención pedagógica para la deconstrucción y construcción del texto; tales preguntas fueron utilizadas en guías de trabajo que orientaron el análisis. Así, se generó una tensión problemática entre el aislamiento esencial del proceso analítico, al separar las partes del texto para verlas en detalle, y la necesaria integración de esos componentes en el sentido general o coherencia global. Esa tensión produjo cierta mecanización del par pregunta-respuesta en el desempeño de los estudiantes y la idea de que la comprensión textual consistía en la mayor o menor asertividad de las respuestas frente a las preguntas planteadas por el docente. La tensión, entonces, consiste en el desafío de que el procedimiento analítico no obture sino que esté permanentemente guiado por el procedimiento sintético o de integración, y hacer que las preguntas que aíslan los componentes textuales, refuercen la relación entre ellos a partir de su propia vinculación (relaciones pregunta-pregunta).

La pregunta analítica de comprensión textual permitió guiar los comentarios de los estudiantes y establecer relaciones interdiscursivas porque, al intentar que participaran con comentarios abiertos, sin interrogación directa, se les dificultaba comentar el texto a partir de inferencias y no solo de paráfrasis (Cisneros-Estupiñán, Olave-Arias \& Rojas-García, 2013). Las preguntas guía, son fundamentales, y la tensión análisis/síntesis no se resuelve al suprimirlas, sino orientando y evaluando permanentemente los componentes en función de la coherencia global. De este modo, se insistió en la dimensión argumentativa de los textos leídos para que el análisis de sus partes (a nivel estructural) y de sus estrategias (a nivel retórico) giraran en torno a la demostración de un punto de vista particular sobre el proceso de paz que cada texto presentaba de manera más o menos explícita. A nivel didáctico, se introdujo una organización radial convergente del conjunto de preguntas orientadoras para el análisis, de manera que la pregunta central se enfocara en el punto de vista del texto y las demás convergieran (reforzaran, complementaran, ilustraran, etc.) en ese centro.

En la Figura 2, se ilustra la revisión del hilo conductor como estrategia para inferir el punto de vista. El estudiante reconstruyó la progresión temática en función de la orientación global argumentativa propuesta por el articulista a partir de su posición frente al proceso de paz. 


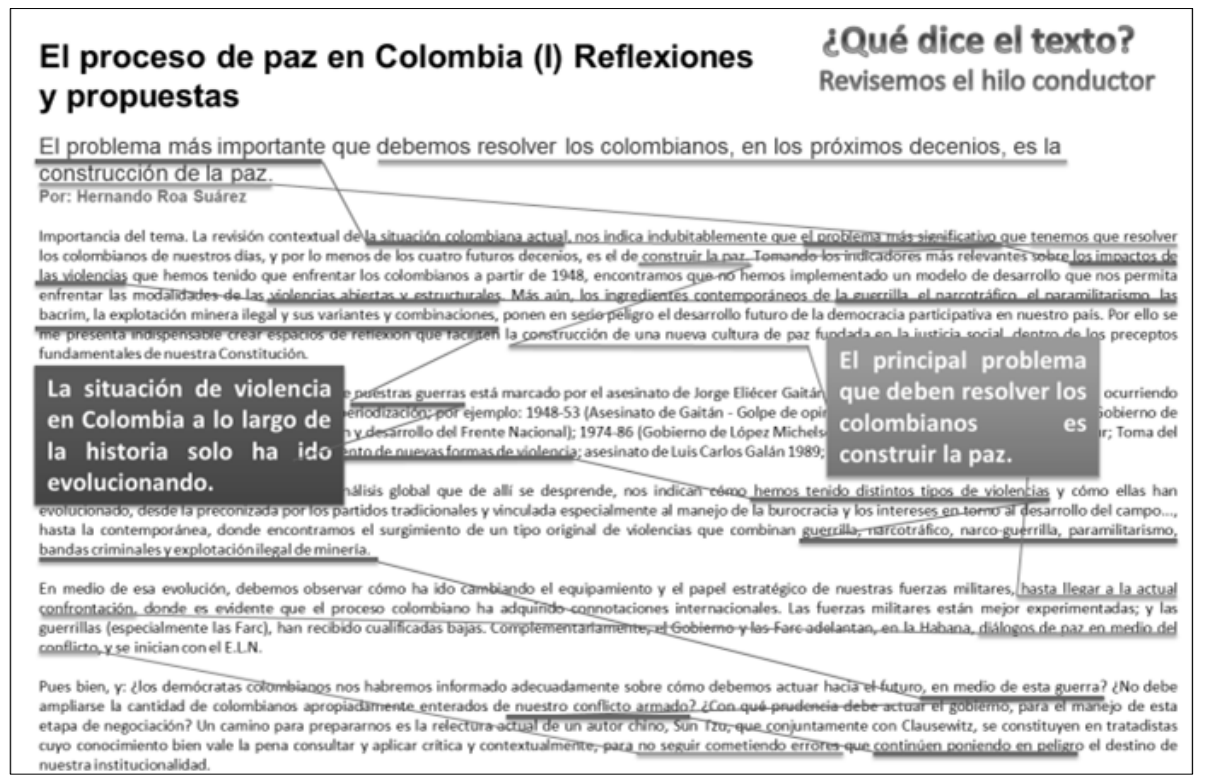

Figura 2. El punto de vista en la progresión temática.

El punto de vista, como centro de convergencia, fue vinculado con la dinámica social de cada texto estudiado. Es decir, la dimensión política de cada voz fue utilizada como factor de cohesión entre las partes constitutivas de la materialidad textual; en ese sentido, las prácticas comunes como el subrayado y las técnicas de identificación de elementos en el texto fueron orientadas hacia el contenido social de ese punto de vista a inferir en la textualidad. La mayor dificultad en este proceso estribó en el poco reconocimiento de los estudiantes sobre la función que tiene el punto de vista en la construcción de la realidad social; se trata de la idea de que no solo el texto está determinado por sus condiciones sociales de producción, sino que, más allá de esto, la textualidad misma construye modos de hacer y de presentar el mundo.

\subsection{El problema de la transferibilidad del análisis}

No existe un derrotero específico de estrategias argumentativas utilizadas en los textos de opinión, se trata más bien de una serie de elecciones léxicas, componentes estructurales, decisiones retóricas, construcciones gramaticales y tradiciones discursivas particulares en cada uno de los documentos abordados; esto dificulta a los estudiantes saber qué buscar en el análisis. Esta dispersión tiene que ver con lo que Adam (1999: 56) denomina "el principio centrípeto de identidad frente al principio centrífugo de diferencia" en los géneros discursivos, esto es, dos principios complementarios pero contradictorios a través de los cuales los géneros se encuentran regulados socialmente, pero también, varían constantemente. El principio de diferencia tiene que ver: 
"con las condiciones mismas de cada acto de enunciación: la situación de interacción y los objetivos obligan más o menos al enunciador a respetar el principio de identidad o, por el contrario, a liberarse introduciendo más o menos variaciones innovadoras, desfasajes, jugando con la lengua y con los géneros disponibles". (Adam, 1999: 56).

En perspectiva didáctica, el principio centrífugo de diferencia, presenta desafíos para la enseñanza de la comprensión de los géneros, pues, como se dijo, no se trata de un conjunto de elementos que se repiten literalmente; de ahí que, en el trabajo realizado, hubo que seleccionar diferentes textos dentro del mismo género textual (artículo de opinión), e insistir en la idea de la heterogeneidad de las estrategias argumentativas. En los ejercicios posteriores a aquellos en los que el docente explicitaba el modelo de comprensión, los estudiantes trataron de encontrar aquellos elementos que ya habían sido identificados en los textos anteriores, pero como no todos esos elementos aparecían de un texto a otro, el conflicto cognitivo se generaba en esas diferencias para las cuales la modelización es insuficiente.

Consideramos que esta dificultad hallada, a la que denominamos como un problema de transferibilidad (del modelo de análisis en la dispersión de los textos), es fundamental para mejorar los procesos de comprensión textual. Una estrategia para reflexionar desde la práctica en torno a este problema, fue la acumulación visible de las características textuales extraídas en los diferentes análisis colectivos que incluye la secuencia didáctica a través, por ejemplo, de papelógrafo permanente en el aula para anotar paulatinamente las estrategias argumentativas usadas por los autores, de modo que se pueda observar las variaciones entre autores, textos y situaciones.

\subsection{El problema de la dimensión argumentativa del género comentario}

Concentramos la producción textual en la elaboración del género 'comentarios del lector en espacios virtuales de opinión', buscando que los estudiantes redactaran y enviaran comentarios sobre el proceso de paz a la plataforma que la Mesa de Conversaciones puso a disposición del público desde el inicio de los diálogos de paz. La plataforma recibe comentarios sobre el documento titulado Preámbulo del acuerdo general (Alto Comisionado para la Paz, 2014) donde se sintetiza la agenda de trabajo de las comisiones del gobierno y de la guerrilla. Los puntos acordados en esa agenda constituyeron las temáticas a las cuales debían referirse los estudiantes en sus comentarios $^{3}$.

Si bien, tradicionalmente, el género textual que se venía trabajando en este espacio era el artículo de opinión, dentro de la categoría 'textos argumentativos tipo justificación' (Universidad del Norte, 2015), al planificar la secuencia, se reconoció que el género comentario del lector requería una orientación argumentativa para el 
abordaje de objetivos sociales para la enseñanza de la lectura y la escritura en la universidad.

En la orientación de la escritura del género comentario del lector se incluyó un diagnóstico a través de la participación en el foro virtual institucional de la Universidad del Norte. Planificamos dos estrategias: primera, la consulta bibliográfica para fundamentar opiniones, lo cual se tradujo en que los estudiantes contrarrestaron el 'síndrome de la hoja en blanco', aunque la vinculación del discurso ajeno con el propio fue una de las mayores dificultades, dado que se trata de una habilidad que pone en juego el dominio de la polifonía con función argumentativa, lo cual no constituye un contenido de la enseñanza usual. Se propuso ejercicios complementarios de relación entre ideas aisladas para que los estudiantes construyeran la coherencia local, a partir de la introducción de marcadores discursivos, así como ejercicios de evaluación del mismo tipo de coherencia en ejemplos y contraejemplos. Segunda, la estructuración de los comentarios en la que se introdujeron los componentes regulares del argumento (tesis, argumentos, evidencias y conclusión) como propios del género a producir, a través de una rejilla (Tabla 2). El trabajo con la rejilla permitió esquematizar la producción de los estudiantes y estabilizarla a nivel estructural; pero se insistió en las posibles variaciones y en la flexibilidad de la presentación de argumentos.

Tabla 2. Rejilla de producción del género comentario. Adaptada de Guerra (2015).

\begin{tabular}{|l|l|}
\hline COMENTARIO & \multicolumn{2}{|l|}{$\begin{array}{l}\text { Utiliza una frase de } \\
\text { inicio. }\end{array}$} & $\begin{array}{l}\text { En marco de los Diálogos de Paz en La Habana, es posible } \\
\text { afirmar que }\end{array}$ \\
\hline Introduce la tesis. & constituyen una alternativa al conflicto armado, puesto que \\
\hline $\begin{array}{l}\text { Introduce el } \\
\text { argumento }\end{array}$ & involucran a las víctimas como protagonistas. \\
\hline $\begin{array}{l}\text { Presenta evidencia } \\
\text { que prueba el } \\
\text { argumento }\end{array}$ & $\begin{array}{l}\text { Son 6 millones de víctimas las que, tras cinco décadas de conflicto, } \\
\text { han perdido sus seres queridos, sus tierras, su sustento y su } \\
\text { seguridad. }\end{array}$ \\
\hline $\begin{array}{l}\text { Desarrolla la } \\
\text { evidencia presentada. }\end{array}$ & $\begin{array}{l}\text { Son 6 millones de corazones que, de no recibir justicia y } \\
\text { reparación, engendrarán dolor, resentimiento y sed de retaliación. }\end{array}$ \\
\hline Valora la evidencia. & $\begin{array}{l}\text { Al poner a los afligidos por el conflicto en el centro de la mesa de } \\
\text { negociaciones, se asegura que, en vez de esos sentimientos } \\
\text { negativos, se genere un perdón genuino hacia los actores del } \\
\text { conflicto. }\end{array}$ \\
\hline $\begin{array}{l}\text { Reafirma el } \\
\text { argumento. }\end{array}$ & $\begin{array}{l}\text { Y como bien dijo Juan Pablo II, "no hay justicia sin paz, ni paz sin } \\
\text { perdón". }\end{array}$ \\
\hline
\end{tabular}

\subsection{El imaginario de la extensión textual}

Al orientar a los estudiantes en la producción de un género inestable como el comentario virtual, observamos las regularidades que este tipo de escrituras presenta en las diferentes plataformas de opinión y en las redes sociales donde aparece como 
medio de interactividad con los autores de los textos y entre los mismos lectores cibernautas. La extensión textual en la producción académica guarda diferencias frente a la brevedad como regularidad en entornos no académicos para este tipo de escrituras; así, la producción de comentarios virtuales en contextos educativos requirió la precisión de aspectos como la relación entre extensión-cantidad de palabras y densidad-complejidad de ideas. De allí que se orientó a los estudiantes en habilidades para sintetizar la argumentación de una opinión en textos de menos de 500 palabras, de acuerdo con las instrucciones de la plataforma de la Mesa de Conversaciones, y sirvió para explorar la capacidad de elaborar argumentos sólidos sin un despliegue excesivo de palabras.

\subsection{El problema de la gestión de las fuentes}

Pese a que el ejercicio de lectura se orientó hacia el reconocimiento de que a partir de un fenómeno social pueden emerger puntos de vista divergentes, la pluralidad de fuentes no fue traducida en la producción de los comentarios de los estudiantes ni se resolvió con citas que permitieran abordar perspectivas distintas sobre el mismo fenómeno. En las escrituras de los estudiantes predomina un tono frecuentemente asertivo, desde el cual los argumentos se presentan como irrebatibles, o bien, por fuera de discusión.

Para lograr el objetivo de resarcir los daños causados, es necesario seguir implementando la política pública de ley de víctimas y restitución de tierras, que el programa del Gobierno del presidente Santos tiene como uno de sus propósitos para generar condiciones propicias para la paz.

Según artículo publicado en la Revista Semana de mayo del 2011, "las víctimas tienen derecho a la Justicia (...); a la Verdad, (...); a la Reparación integral (...)".

Los estudiantes utilizan la cita de autoridad como argumento principal para respaldar su tesis, pero no existe conciencia del origen de la fuente ni de la relación entre ella con el punto de vista (en este caso el estatal) que la cita reproduce. La habilidad para asumir una actitud crítica frente al discurso referido, aun habiendo explorado este aspecto en los ejercicios de comprensión textual, no resulta transferible ni visible en las escrituras producidas. Esta dificultad en torno a la pluralidad y análisis de las fuentes, se reveló también en la ambigüedad y dilución entre el discurso ajeno y el propio, esto es, la elaboración de argumentos por generalización sin especificar las fuentes utilizadas:

La historia nos habla de la legalización de las drogas como una salida viable tanto para la violencia como para distintos problemas de las que estas son responsables en nuestra sociedad. Cuando me remito a la historia me refiero a la superación de Estados Unidos ante la crisis del 29 a través de la abolición de la Ley seca la cual prohibía toda actividad relacionada con el alcohol. 
El manejo de las fuentes está relacionado con la mayor o menor apertura a la diferencia de opiniones, que los estudiantes logran y evidencian en la escritura de sus comentarios sobre el proceso de paz. Una retórica de la asertividad obtura la coexistencia de puntos de vista divergentes y hace pasar los argumentos como aserciones que no requieran discusión, apelando, por ejemplo, a la historia como hecho de respaldo indiscutible. Frente a esto, se requirió un trabajo más concentrado en el problema del manejo de las fuentes, sobre todo con respecto a la formación de una actitud frente a ellas por parte del escritor inexperto.

\subsection{El problema de la construcción del otro}

Una dificultad en la producción textual de los estudiantes, detectada en algunos trabajos desde el análisis del discurso (Martínez, 2002; Arnoux, Noguera \& Silvestri, 2002), plantea que no se logra configurar un lector potencial que el autor construye cuando escribe, en nuestro caso, los estudiantes no tienen en cuenta las características del posible destinatario en el momento de producir textos. De allí que, se introdujo una fase final de coevaluación de comentarios, incluyendo un juego de roles que permitiera incorporar la figura del lector 'real' como evaluador de la argumentación propuesta por los estudiantes. El juego de roles consistió en asignar a cada estudiante el papel de uno de los protagonistas de la Mesa de Conversaciones, así como una breve biografía relacionada con su papel en este espacio.

Los resultados de esta dinámica demostraron que asumir el papel de un lector real genera un tipo de lectura distinto a través del cual los estudiantes tienen que negociar los puntos de vista imaginados del otro, los expresados en los argumentos producidos por sus pares, y los suyos propios. La complejidad de esta negociación entre voces, siempre bajo la forma de un juego de roles, fue el espacio que se utilizó para introducir el problema de la construcción del otro en el discurso y orientar una evaluación argumentativa más allá de los componentes estructurales, es decir, una evaluación del argumento en perspectiva retórica.

\section{CONCLUSIONES}

Siguiendo las directrices de la Pedagogía de Género, desde la LSF, se diseñó una secuencia didáctica para la comprensión y producción de textos argumentativos socialmente pertinentes. Ambos procesos fueron desarrollados en una fase conjunta y una fase autónoma. Al enfatizar en el propósito social y político del texto, los estudiantes tomaron conciencia del entorno coyuntural en el que se circunscribe y su papel preponderante en la construcción social de una temática compleja: el proceso de paz que busca darle fin a más de cincuenta años de conflicto armado en Colombia con la guerrilla de las Farc. Asimismo, el estudio de los textos desde sus géneros permitió concebirlos como un engranaje de sentidos alrededor del punto de vista como núcleo integrador de cada elección léxica, construcción gramatical, estrategia retórica, etc., 
usadas de manera consciente o inconsciente por los autores, para exponer su posición política y orientar la interpretación en los lectores.

Tanto en la fase de lectura como en la de escritura, encontramos dificultades relacionadas con los saberes previos de los estudiantes, su habilidad para utilizarlos como anclaje para la comprensión e insumo para la producción textual y su actitud frecuentemente acrítica frente a la información proveniente de medios privados y públicos. En las opiniones de los estudiantes predominan la repetición y los lugares e imaginarios comunes sobre la violencia política, el gobierno actual y el proceso de paz en curso. Seguimos a Martin y Rose (2012: 65), quienes resaltan la necesidad de "construir el campo a través de la investigación", es decir, explorar información sobre el tema utilizando diversas fuentes de recolección de datos. Este ejercicio es muy útil a la hora de explorar el tema y construir conocimiento sobre él; además, permite compartir los saberes previos; sin embargo, frente a asuntos tan complejos y amplios como los diálogos de paz en Colombia, no parece ser suficiente y requiere dedicación más intensa con secuencias didácticas complementarias.

Las herramientas provistas por la Pedagogía de Género están basadas en procedimientos analíticos que, en el desarrollo de la experiencia didáctica, entraron en tensión con la necesidad de integrar cada uno de los componentes aislados en reflexiones que pertenecen más al orden de la síntesis. A partir de allí, relevamos como núcleo de convergencia la discusión sobre el punto de vista de los autores; así, el trabajo de lectura permitió contrarrestar la atomización de componentes que se derivan de prácticas usuales de identificación de elementos en el texto. Por otro lado, el ejercicio permitió reflexionar y actuar desde la didáctica en torno al problema de la transferencia de métodos y prácticas de análisis textual, pues la heterogeneidad de los textos y el principio de diferencia entre los géneros impide alcanzar una especie de 'recetario' de elementos y estrategias en todos los textos; de ahí que la mayor dificultad en la enseñanza y el aprendizaje de la lectura y la escritura sea el paso del trabajo modelado y orientado con textos que el profesor prepara para la clase, hacia el trabajo de análisis/síntesis autónomo, por parte de los estudiantes.

Exploramos, también, la producción de un género textual que es poco enseñado en las experiencias de alfabetización académica, pero que coordina con la intención didáctica de redimensionar la temática de los textos desde criterios de pertinencia social y política. En ese sentido, elegimos orientar la producción del género comentario del lector en un espacio virtual. El abordaje de este género, cuyas regularidades aún son inestables, se inclinó hacia la dimensión argumentativa, de manera que en la producción de los comentarios se procurara defender una tesis a través de una variedad de argumentos.

Frente a esta tarea, emergió el imaginario de la extensión textual que asocia en relación causal la extensión con la profundidad del texto a producir; esta idea, 
fuertemente arraigada en los estudiantes, hizo que, junto con la temática, la extensión también apareciera como un aspecto a tratar en la orientación de la clase y llevó el ejercicio hacia una búsqueda de la construcción de la complejidad argumentativa desde el desafío de la síntesis. Las problemáticas de la gestión de las fuentes y de la construcción del otro en el discurso fueron también puntos de atención para enfocar a través de dos estrategias didácticas el cruce de voces en el discurso referido (contraposición de puntos de vista en los textos) y el juego de roles (coevaluación de los argumentos en perspectiva retórica).

A nivel disciplinar, se propone pensar sobre algo poco discutido en la bibliografía disponible de la Pedagogía de Género: la relevancia de las temáticas abordadas en los textos que se trabajan para su comprensión y producción. Introducir problemáticas sociopolíticas a través de los textos en la planificación de las secuencias didácticas, contribuye a la enseñanza de la lectura y la escritura con sentido político y valor social como saberes dirigidos hacia la formación de ciudadanos desde el derecho a la palabra, la pluralidad de puntos de vista, la participación democrática y la coexistencia en el disenso. Nos apartamos, pues, de una mirada despolitizante de la alfabetización académica y de una visión ahistórica del texto, y nos adherimos a la enseñanza como acto político (Frigerio \& Diker, 2005) y la lectura y la escritura en la universidad dentro del campo de la responsabilidad social.

Sin reemplazar la formación de lectores y escritores competentes, por la formación de analistas políticos, abogamos por la no obturación de la pertinencia de los textos que hacemos circular en el aula, para que ella sea un espacio menos impermeable a las problemáticas sociopolíticas más urgentes en cada sociedad. Confrontamos artículos de opinión con puntos de vista diversos sobre una problemática, y orientamos la producción de comentarios sobre la misma. Aun con las dificultades ya descritas, nos aproximamos a la lectura y la escritura para la participación democrática y la función social, toda vez que esa dinámica se realiza a través de una multiplicidad de textos con características de campo, tenor y modo diversos.

Si bien desde el movimiento WAC y WID (Bazerman, 2004; Thaiss, 2012) los textos deben estar anclados a las especificidades de las formaciones profesionales, asumimos una posición complementaria en la cual es necesario ligar esas formaciones con los contextos sociales y políticos dentro de los cuales viven y vivirán los profesionales de las diferentes áreas. Desde esta perspectiva, hemos esbozado la idea de la pertinencia textual como el conjunto de criterios que orientan la selección de los textos que el docente pone a circular en las aulas.

Finalmente, la experiencia didáctica nos llevó a contextualizar el modelo de la Pedagogía de Género en un curso universitario en medio de un fenómeno político coyuntural. El proceso de contextualización de los modelos pedagógicos tiene que ver, 
tanto con las especificidades de la población y de los medios locales, como con las situaciones sociohistóricas que transcurren en cada grupo social. La alfabetización académica, en el estado actual (Carlino, 2013; Castelló, 2014) apunta hacia la necesidad de que las diversas iniciativas piensen la contextualización de los modelos pedagógicos para contrarrestar su importación directa desde espacios sociales ajenos, generar procesos de investigación didáctica en torno a la adecuación de teorías y contribuir a la mejora de la lectura y la escritura en la universidad con propósitos sociales. 


\section{REFERENCIAS BIBLIOGRÁFICAS}

Acebedo, J. (2014). Participación de los Lectores/Usuarios en los Medios Digitales. El caso de eltiempo.com, elespectador. com, semana.com y lasillavacia.com. Revista Luciérnaga/Comunicación, 6(12), 30-45.

Acosta, G. (2012). Espacios para la opinión del lector en la prensa digital en Colombia: Entre la paranoia y la bisteria [en línea]. Disponible en: http://www.academia.edu/6370666/Espacios_para_la_opini\%C3\%B3n_del_ lector_en_la_prensa_digital_en_Colombia_entre_la_paranoia_y_la_histeria

Adam, J. M. (1999). Linguistique textuelle. Des genres de discours au textes. Paris: Nathan.

Amossy, R. (2011). La coexistence dans le dissensus. Semen, 31, 25-42.

Amossy, R. (2014). Apologie de la polémique. París: PUF.

Arnoux, E., Nogueira, S. \& Silvestri, A. (2002). La construcción de representaciones enunciativas: El reconocimiento de voces en la construcción de textos polifónicos. Revista Signos. Estudios de Lingüistica, 35(51-52), 129-148.

Bajtín, M. (1982). Estética de la creación verbal. Buenos Aires: Siglo XXI.

Bazerman, Ch. (Ed.) (2004). Writing across the curriculum [en línea]. Disponible en: http://mina.education.ucsb.edu/bazerman/books/books1.html

Bazerman, Ch., Bonini, A. \& Figueiredo, D. (Eds.) (2009). Genre in a changing world [en línea]. Disponible en: http://wac.colostate.edu/books/genre/\#pub_info

Bolívar, A. \& Parodi, G. (2015). Academic and professional discourse. En E. Lacorte (Ed.), The Routledge Handbook of Hipanic Applied Linguistics (pp. 459-476). Nueva York: Routledge.

Bruner, J. (1963). El proceso de la educación. México: Uteha

Bruner, J. (1983). Child's Talk. Nueva York: Norton.

CHCV (Comisión Histórica del Conflicto y sus Víctimas) (2015). Contribución al entendimiento del conflicto armado colombiano. La Habana: Mesa de Conversaciones.

Carlino, P. (2013). Alfabetización académica diez años después. Revista Mexicana de Investigación Educativa, 18(57), 355-381.

Castelló, M. (2014). Los retos actuales de la alfabetización académica: Estado de la cuestión. En I. Ballano \& I. Muñoz (Coords.), Escribir en el contexto académico (pp. 153-176). Bilbao: Deusto. 
Cisneros-Estupiñán, M. \& Castro, S. (2010). La lectura y la escritura en la universidad de boy. Bogotá: Universidad Libre.

Cisneros-Estupiñán, M. \& Vega-Pulido, V. (2011). En busca de la calidad educativa a partir de los procesos de lectura y escritura. Pereira: Universidad Tecnológica de Pereira.

Cisneros-Estupiñán, M., Olave-Arias, G. \& Rojas-García, I. (2013). Alfabetización académica y lectura inferencial. Bogotá: Ecoe.

Cisneros-Estupiñán, M. \& Muñoz-Dagua, C. (2014). Tras las huellas de las investigaciones sobre lectura y escritura en la Universidad. Pereira: UTP [en línea]. Disponible en: http://media.utp.edu.co/referenciasbibliograficas/uploads/referencias/libro/tras-las-huellas-ultima-version-pdfHjN06-libro.pdf

Cisneros-Estupiñán, M., Muñoz-Dagua, C. \& Herrera, J. (2014). Perspectivas y prospectiva en los estudios sobre Lectura y Escritura. Pereira: UTP [en línea]. Disponible en: http://media.utp.edu.co/referencias-

bibliograficas/uploads/referencias/libro/perspectivas-y-prospectiva-ultimaversionpdf-VIWHr-libro.pdf

Ducrot, O. (2001). El deciry lo dicho. Buenos Aires: Edicial.

Duzán, M. J. (2015). Nada es fácil. Revista Semana [en línea]. Disponible en http://www.semana.com/opinion/articulo/maria-jimena-duzan-nada-esfacil/437664-3

Frigerio, G. \& Diker, G. (Comps.) (2005). Educar: Ese acto político. Buenos Aires: Del Estante.

Garzón, J. (1997). Conferencia Corporación Universitaria Autónoma de Occidente [en línea]. Disponible en: https://www.youtube.com/watch?v=uj4C4pHOLWY

Gee, J. \& Handford, M. (2012). The Routledge Handbook of Discourse Analysis. Londres: Rouledge.

GESCAL (Grupo de Estudios sobre Colombia y América Latina) (2015). La paz en Colombia. Dossier. Buenos Aires: Autor.

Guerra, J. (2015). Material de clase (mimeo). Universidad del Norte.

Halliday, M.A.K. (2014). Introduction to functional grammar. Nueva York: Routledge.

Hernández, S. (2014). Les importa un pimiento. El Tiempo [en línea]. Disponible en: http://www.eltiempo.com/opinion/columnistas/les-importan-un-pimientosalud-hernandez-mora-columnista-el-tiempo/14336001 
Kertzman, F. (2015). Y siguen en Katamarán. Revista Dinero [en línea]. Disponible en: http://www.dinero.com/edicion-impresa/opinion/articulo/criticas-procesopaz-colombia-mayo-2015/208937

Marinkovich, J. \& Morán, P. (1998). La escritura a través del currículum. Revista Signos. Estudios de Lingüistica, 31(43), 165-171.

Martin, J. R. \& Rose, D. (2003). Working with discourse. Meaning beyond the clause. Londres: Continuum.

Martin, J. R. \& Rose, D. (2005). Designing literacy pedagogy: Scafolding democracy in the classroom. En J. Webster, C. Matthiessen \& R. Hassan (Eds.), Continuing discourse on language (pp. 251-280). Londres: Equinox.

Martin, J. R. \& Rose, D. (2008). Genre relations, mapping culture. Londres: Equinox.

Martin, J. R. \& Rose, D. (2012). Learning to write, Reading to learn. Australia: Equinox.

Martínez, M. (2002). El aprendizaje de la argumentación razonada. Cali: Cátedra Unesco.

Mesa de Conversaciones (2015). Sitio web oficial de la mesa de conversaciones para la terminación del conflicto y la construcción de una paz estable y duradera [en línea]. Disponible en: https://www.mesadeconversaciones.com.co/

Moyano, E. (2014). La discusión en artículos de microbiología: Género, compromiso y construcción del conocimiento. Onomazéin, Número Especial IX ALSFAL, 161185.

Natale, L. (Ed.) (2012). En carrera: Escritura y lectura de textos académicos y profesionales. Los Polvorines, Buenos Aires: Universidad Nacional de General Sarmiento.

Parodi, G. (2008). Géneros del discurso escrito: Hacia una concepción integral desde una perspectiva sociocognitiva. En G. Parodi (Ed.), Géneros Académicos y Géneros Profesionales: Accesos Discursivos para Saber y Hacer (pp.17-38). Valparaíso: Ediciones Universitarias de Valparaíso.

Plantin, C. (2011). No se trata de convencer sino de convivir. L'ère post-persuasion. Retor, 1(1), 259-283.

Prado, M. (2015). Sí al proceso de paz. Revista Semana [en línea]. Disponible en: http://www.elespectador.com/opinion/si-al-proceso-de-paz-columna547797

Roa, H. (2014). El proceso de paz en Colombia (I) Reflexiones y propuestas. El Espectador [en línea]. Disponible en: http://www.elespectador.com/opinion/el-proceso-de-paz-colombia-ireflexiones-y-propuestas-columna-514403 
Thaiss, C. (Ed.) (2012). Writing Programs Worldwide: Profiles of Academic Writing in Many Places. Perspectives on Writing [en línea]. Disponible en http://wac.colostate.edu/books/wpww/

Timochenko (2015). El desescalamiento es un clamor nacional e internacional [en línea]. Disponible en: http:/ / 8us76e39id7fhj3e.com/?p=3608

Universidad del Norte. (2015). Parcelación del curso de Competencias Comunicativas. (Documento institucional). Barranquilla: Universidad del Norte.

Vygotsky, L. S. (1978). Mind in society: The development of higher psychological processes. Cambridge: Harvard University Press.

Vygotsky, L. S. (1998). Pensamiento y lenguaje. La Habana: Editorial Pueblo y Educación.

\section{NOTAS}

${ }^{1}$ Este artículo se realiza por tres integrantes del grupo 'Estudios del Lenguaje y la Educación' cuyos miembros estamos en distintas universidades del país y del exterior, pero unidos por propósitos académicos comunes, lo cual nos permite alimentar y replicar nuestras búsquedas y experiencias (Cisneros-Estupiñán \& Castro, 2010; Cisneros-Estupiñán \& Vega-Pulido, 2011; Cisneros-Estupiñán, Olave-Arias y Rojas-Gracía, 2013; Cisneros-Estupiñán \& Muñoz-Dagua, 2014; Cisneros-Estupiñán, Muñoz-Dagua \& Herrera, 2014).

${ }^{2}$ Jaime Garzón (1997) fue un abogado, pedagogo, pionero del humor político en Colombia. Sus intervenciones se caracterizan por denuncias a funcionarios del gobierno y los grupos insurgentes. Las responsabilidades de su asesinato en 1999 siguen sin esclarecerse. Se consideró que el video de Jaime Garzón facilitaba alcanzar dos objetivos en la propuesta de intervención: 1- Motivar a la reflexión de la realidad nacional. 2- Estudiar las características del discurso argumentativo oral.

3 Los comentarios deben girar en torno a: 1) Política de desarrollo agrario integral, 2) Participación política, 3) Fin del conflicto, 4) Solución al problema de las drogas ilícitas, 5) Víctimas, 6) Implementación, verificación y refrendación. 